\title{
WATER DISTRIBUTION SYSTEM DESIGN \\ UNDER UNCERTAINTIES
}

K.E. Lansey, N. Duan, L.W. Mays and Y.K. Tung

1989

Journal Article

WWRC - 89-18

In

Journal of Water Resources

Planning and Management

Volume 115

\author{
K.E. Lansey \\ School of Civil Engineering \\ Oklahoma State University \\ Stillwater, Oklahoma \\ N. Duan \\ L.W. Mays \\ Center for Research in Water Resources \\ University of Texas \\ Austin, Texas \\ Y.K. Tung \\ Wyoming Water Research Center \\ University of Wyoming \\ Laramie, Wyoming
}




\title{
Water Distribution System Design UNDER UNCERTAINTIES
}

\author{
By Kevin E. Lansey, ${ }^{1}$ Associate Member, ASCE, Ning Duan, ${ }^{2}$ \\ Larry W. Mays, ${ }^{3}$ Member, ASCE, and Yeou-Koung Tung, \\ Associate Member, ASCE
}

\begin{abstract}
A chance constrained model is presented for the minimum cost design of water distribution networks. This methodology attempts to account for the uncertainties in required demands, required pressure heads, and pipe roughness coefficients. The optimization problem is formulated as a nonlinear programming model which is solved using a generalized reduced gradient method. Details of the mathematical model formulation are presented along with example applications. Results illustrate that uncertainties in future demands, pressure head requirements, and pipe roughness can have significant effects on the optimal network design and cost.
\end{abstract}

\section{INTRODUCTION}

There is currently no universally accepted definition or measure of the reliability of water distribution systems. In general, reliability is defined as the probability that a system performs its mission within specified limits for a given period of time in a specified environment. Over the past two decades, there have been many models developed for the analysis and the minimum cost design of water distribution networks (e.g., Alperovits and Shamir 1977; Quindry et al. 1981; Morgan and Goulter 1986; Lansey and Mays 1987). Only a very few models have been reported that attempt to consider the reliability of the water distribution network and the various components. Coals and Goulter (1985) presented three approaches by which the probability of failure of individual pipes can be related to a measure of the overall system reliability in a linear programming minimum cost design procedure. No models explicitly consider the uncertainties in demands, pressure heads, and pipe roughness.

The real issue of water distribution system reliability concerns the ability of the system to supply the demands at the nodes or demand points within the system at required minimum pressures. The conventional design process for water distribution systems is a trial and error procedure that attempts to find a design that represents a least-cost solution that can satisfy demands. These trial and error methods make no attempt to analyze or define any reliability aspects of the designed system and have no guarantee that the

\footnotetext{
'Asst. Prof., School of Civ. Engrg., Oklahoma State Univ., Stillwater, OK 74078; formerly, Res. Asst., Dept. of Civ. Engrg., The Univ. of Texas, Austin, TX 78712.

${ }^{2}$ Res. Engr., Chinese Res. Acad. of Envir. Sci., Beijing, Peoples Republic of China; formerly Res. Asst., Center for Res. in Water Resour., The Univ. of Texas, Austin, TX.

${ }^{3}$ Dir., Center for Res. in Water Resour. and Engrg., Foundation Endowed Prof., Dept. of Civ. Engrg., The Univ. of Texas, Austin, TX.

${ }^{4}$ Assoc. Prof., Dept. of Statistics, and Wyoming Water Res. Center, Univ. of Wyoming, Laramie, WY 82071.

Note. Discussion open until February 1, 1990. To extend the closing date one month, a written request must be filed with the ASCE Manager of Journats. The manuscript for this paper was submitted for review and possible publication on October 27, 1987. This paper is part of the Journal of Water Resources Planning and Management, Vol. 115, No. 5, September, 1989. OASCE, ISSN 0733-9496/89/ $0005-0630 / \$ 1.00+\$ .15$ per page. Paper No. 23875
} 
resulting system is a minimum cost system. The resulting system design is not based upon consideration of the various design uncertainties.

Mays and Cullinane (1986) presented a review of methods that can be used to define the component reliabilities for the various components of a water distribution system. These methods are based upon using time-to-failure and time-to-repair data for the various components of the water distribution system to define reliability and availability. Su et al. (1987) presented a procedure for modeling reliability in water distribution network design that more realistically considers reliability. That is, the reliability is defined in terms of the ability of the system to supply the demands at the nodes or demand points within the system at or above minimum pressure heads. The model uses component failure rates to compute component failure probabilities which are then used to define nodal and system reliabilities. A minimum cut-set method was used in the nodal and system reliability determination. The key issue in this approach was to relate failure probability of the pipes to meeting specified demands (flow rates) at or above minimum pressure heads at the demand nodes. The procedure was linked to a nonlinear programming optimization model to determine a minimum cost water distribution system considering nodal and system reliabilities as constraints.

Water distribution systems are designed to service consumers over a long period of time. Because the number and types of future consumers are impossible to define with any accuracy, the projected future required demands and required pressure heads for design are very uncertain. Another uncertain parameter in the design of a system is the system capacity. The capacity is affected by corrosion of pipes, deposition in pipes, even the physical layout and installation of the system which has a marked effect on the carrying capacity. The change in system capacity can be reflected in the roughness coefficient of the pipes (Hudson 1966). Since the impact of the different mechanisms that decrease system capacity is not known, there is uncertainty in the projections of the coefficients of roughness. The variation of roughness is illustrated in the work by Hudson who compared the Hazen-Williams roughness coefficient for seven U.S. cities as a function of age of pipe.

To the investigators' knowledge, no models have been developed for the minimum cost design of water distribution networks that directly consider the uncertainties in demand requirements, pressure head requirements, and roughness coefficients. Previous models considered uncertainties in delivering flows and pressure heads during pipe failures. There have been many works reported in the literature that deal with uncertainties in water supply forecasting and modeling; however, very little work has been performed in developing a model that directly considers the uncertainties of required demand and other system parameters in the design of water distribution systems.

The objective of this paper is to present a methodology which incorporates the uncertainties in required demands, required pressure heads and roughness coefficients in the design of water distribution systems. This model is based upon the premise that water distribution sytems are designed using specified demands, pressure heads, and roughness coefficients that are basically uncertain parameters that vary considerably with time. The required demand, $Q$, and the required pressure head are dependent upon consumer need whereas the roughness coefficient depends upon other factors. It can be argued that the demands for various demand nodes are not independent and the $C$ 's for 
various pipes are not independent. Obviously, the $C$ values are affected by age, corrosion, deposition, etc., and all pipes may be affected similarly. In theory, the presence of any correlation among variables can be included in the analysis in a straightforward manner. However, because of the lack of any available data to compute co-variances and for the sake of simplicity, the $Q, H$, and $C$ values, in the present study, are each assumed to be independent.

The methodology is presented through the formulation of an optimization model for the design of water distribution systems. This optimization model is based upon a nonlinear chance-constrained formulation and can be solved using generalized reduced gradient methods, such as GRG 2 by Lasdon and Waren (1984). Details of the mathematical model are given in addition to examples to illustrate the methodology.

\section{Model Development}

The basic optimization model for water distribution system design can be stated in general form as:

Min. Cost $=\sum_{i, j \in M} f\left(D_{i, j}\right)$

subject to the following constraints

$\sum_{j} q_{i, j}=Q_{j} \quad j=1, \ldots, J$ (nodes)

$\sum_{i, j \in n} h_{n}=0 \quad n=1, \ldots, N$ (loops)

$H_{j} \geq H_{j} \quad j=1, \ldots, J$

$D_{i, j} \geq 0$

For purposes of discussion and simplicity of the model development, only pipes are considered for the design; however, pumps, valves, and other special hydraulic appurtanences can be included.

The objective function is to minimize cost as a function of the diameter $D_{i, j}$, for the set of possible links, $M$, connecting nodes $i$ and $j$ in the network. Constraint Eq. 2 is the continuity equation used to satisfy demand at each node in which $q_{i, j}$ is the flow rate in the pipe connecting nodes $i$ and $j$, and $Q_{j}$ is the external demand at node $j$. This constraint is written for each node $j$ in the network. Constraint Eq. 3 states that the sum of the head losses, $h_{n}$, around each loop $n=1, \ldots, N$ is equal to zero. Eq. 4 defines the minimum requirement, $H_{j}$, on the pressure head, $H_{j}$, at each node.

The discharge $q_{i, j}$ in each pipe connecting nodes $i$ and $j$ can be expressed using the Hazen-Williams equation, so that Eq. 2 can be expressed as

$\sum_{j} K \cdot C_{i, j}\left[\frac{H_{i}-H_{j}}{L_{i, j}}\right]^{0.54} D_{l, j}^{2.63}=Q_{j}$

in which $C_{i, j}=$ the Hazen-Williams roughness coefficient; $H_{i}$ and $H_{j}=$ the pressure heads at nodes $i$ and $j$, respectively; $L_{i j}=$ the length of the pipe connecting nodes $i$ and $j$; and $D_{i, j}=$ the pipe diameter of the pipe connecting 
nodes $i$ and $j$, and $K$ is a conversion factor for units. Substituting the HazenWilliams equation into Eq. 2 makes Eq. 3 unnecessary since it is satisfied automatically as shown by Jeppson (1976). The constraint set for the deterministic model now consists of Eqs. 4, 5, and 6.

Considering for design purposes that future demands, $Q_{j}$, minimum pressure head requirements, $H_{j}$, and pipe roughness coefficients, $C_{i, j}$, are uncertain, they are considered as independent random variables from the viewpoint of design. The chance constrained formulation of the model can be expressed as:

Min. Cost $=\sum_{i, j \in M}\left[f\left(D_{i, j}\right)\right]$

subject to

$P\left[\sum_{j} K \cdot C_{i, j}\left(\frac{H_{1}-H_{j}}{L_{i, j}}\right)^{0.54} \cdot D_{i, j}^{2.63} \geq Q_{j}\right] \geq \alpha_{J} \quad j=1, \ldots, J$

$P\left(H_{j} \geq H_{j}\right) \geq \beta_{j} \quad j=1, \ldots, J$

$D_{i, J} \geq 0$.

The objective function Eq. 7 is expressed in terms of minimizing the costs. Constraint Eq. 8 is expressed as the probability, $P($ ), of satisfying demands, i.e., that demands are equalled or exceeded with probability level $\alpha_{j}$. Similarly, constraint Eq. 9 expresses the probability of the minimum pressure head being satisfied, i.e. the pressure heads equal or exceed the minimum pressure head with probability level $\beta_{j}$. In general, the values of the constraint performance reliabilities $\alpha_{j}$ and $\beta_{j}$ can be specified and manipulated to consider the effect of uncertainty.

\section{Deterministic Form of Chance Constrained. Model}

The above model Eqs. 7-10, can be transformed to a deterministic form using the concept of the cumulative probability distribution. This model is based upon the premise that the required demands, pressure head requirements, and roughness for design purposes are designed for uncertain future conditions of the system. Although the theory is general and the variables may follow any distribution, the demands, $Q_{j}$, pressure heads, $H_{j}$, and roughness coefficients, $C_{i, j}$, are assumed to be normal random variables, with means, $\mu$, and standard deviations, $\sigma$, expressed as:

$$
\begin{aligned}
& Q \sim N\left(\mu_{Q}, \sigma_{Q}\right) \\
& H \sim N\left(\mu_{H}, \sigma_{H}\right)
\end{aligned}
$$

and

$C \sim N\left(\mu_{C}, \sigma_{C}\right)$

Constraint Eq. 9 can be written in terms of the standardized variable as

$P\left[\frac{H_{j}-\mu_{H J}}{\sigma_{H J}} \leq \frac{H_{j}-\mu_{H J}}{\sigma_{H J}}\right] \geq \beta_{j}$

and more simply, can be expressed as, 
$\phi\left[\frac{H_{j}-\mu_{H j}}{\sigma_{H j}}\right] \geq \beta_{j}$

in which $\phi$ is the cumulative distribution function. Under the assumption of normality, $\phi[]$ is the standard normal distribution function.

The deterministic form of constraint Eq. 9 can then be expressed as

$H_{j}-\mu_{H j} \geq \sigma_{H j} \phi^{-1}\left(\beta_{j}\right)$

Because $\mu_{H j}, \sigma_{H j}$ and $\beta_{j}$ are all specified, this constraint can be written as a simple bound constraint

$H_{j} \geq \mu_{H j}+\sigma_{H j} \phi^{-1}\left(\beta_{j}\right)$

Similarly, constraint Eq. 8 can also be expressed in a deterministic form. The first step is to rewrite the constraint in the following form:

$P\left[\sum_{i} K \cdot C_{i, j}\left(\frac{H_{i}-H_{j}}{L_{i, j}}\right)^{0.54} D_{i, j}^{2.63}-Q_{j} \leq 0\right] \leq 1-\alpha_{j} \ldots \ldots \ldots \ldots \ldots$

Because both $Q_{j}$ and $C_{i, j}$ are considered normal random variables and are assumed statistically independent, the term on the left side of the inequality,

$W_{j}=\left[\sum_{i} K \cdot C_{i, j}\left(\frac{H_{i}-H_{j}}{L_{i, j}}\right)^{0.54} D_{i, j}^{2.63}-Q_{j}\right]$

is also a normal random variable with mean

$\mu_{w j}=\sum_{i} K \cdot \mu_{c, i, j}\left[\frac{H_{i}-H_{j}}{L_{i, j}}\right]^{0.54} D_{i, j}^{2.63}-\mu_{Q j}$

and standard deviation

$\sigma_{w j}=\left\{\sum_{i}\left[K\left(\frac{H_{i}-H_{j}}{L_{i, j}}\right)^{0.54} D_{i, j}^{2.63}\right]^{2} \sigma_{c, i, j}^{2}+\sigma_{Q j}^{2}\right\}^{1 / 2}$

Constraint Eq. 18 can now be written as

$P\left[\frac{W_{j}-\mu_{w j}}{\sigma_{w j}} \leq \frac{0-\mu_{w j}}{\sigma_{w j}}\right] \leq 1-\alpha_{j}$

which can be simplifed to

$\phi\left[\frac{-\mu_{w j}}{\sigma_{w j}}\right] \leq 1-\alpha_{j}$

The deterministic form of constraint Eq. 18 is then;

$-\frac{\mu_{w j}}{\sigma_{w j}} \leq \phi^{-1}\left(1-\alpha_{j}\right)$

in which $\mu_{w j}$ and $\sigma_{w j}$ are defined by Eqs. 20 and 21 , respectively. If the standard deviations $\sigma_{Q}, \sigma_{H}$, and $\sigma_{C}$ are equal to zero, the parameters are known with certainty.

The deterministic formulation of the chance constrained model is ex- 
pressed by the objective function and constraint Eqs. 24, 17, and 10 as:

Min. Cost $=\sum_{i, j \in m} f\left(D_{i, j}\right)$

subject to

$-\frac{\mu_{w j}}{\sigma_{w j}} \leq \phi^{-1}\left(1-\alpha_{j}\right)$

$H_{j} \geq \mu_{H j}+\sigma_{H j} \phi^{-1}\left(\beta_{j}\right)$

$D_{i, j} \geq 0$

The model is a nonlinear programming problem in which Eqs. 17 and 10 are treated as simple bound constraints. Nonlinearity arises from the expressions for $\mu_{w}$ and $\sigma_{w}$, and for the objective function. Eq. 17 is a simple bound because the right-hand side $\mu_{H j}+\sigma_{H j} \phi^{-1}\left(\beta_{j}\right)$ is known as previously discussed. Constraint Eq. 24 expresses the relationship for the decision variables $H_{j}$ and $D_{i, j}$. The nonlinear problem consists of one nonlinear constraint (Eq. 24) for each node and a simple bound for each decision variable, i.e., one for each pipe link and one for each node. The total number of decision variables is the sum of the number of nodes (number of $H_{j}$ ) and the number of pipe links (number of diameters).

$H$ and $D$ are actually functions of the random variables $Q_{j}, H_{j}$, and $C$; therefore, they should also be considered as random variables. However, through the use of the zero order decision rule for chance-constrained programming (Charnes and Cooper 1963; Charnes and Sterdy 1966), $H$ and $D$ are not considered as random variables. If $H$ and $D$ were considered as random variables, the model formulated herein would be unsolvable. Most chanceconstrained programming applications found in the literature make implicit use of the zero-order decision rule but do not explicitly mention it.

\section{Solution TeChNique}

The above deterministic model formulation of the chance constrained model is nonlinear because of the nonlinear objective function and nonlinear constraints (24). A generalized reduced gradient code, GRG2, by Lasdon and Waren (1984) was used to solve the deterministic form of the chance constrained model (Eqs. 7, 24, 17, and 10). GRG2 requires a user-supplied subroutine GCOMP for the purpose of computing the constraint and objective function values. GCOMP can also be used to read in initial values of any user-required constants. GRG2 is a modular program written to provide dynamic memory allocation with all arrays set up as portions of one large main array so that redimensioning of arrays is never required. Each call to subroutine GCOMP is a function evaluation to compute each constraint and objective function and their gradients for a set of decision variables. Each time the constraint set and gradients are evaluated with a new set of decision variables (pipe sizes), the flow direction is checked.

Generalized reduced gradient methods such as GRG2 require an initial solution to start the optimization search. GRG2 does have the option of using an initial solution provided by the user or to start from an arbitrary solution, as determined by the lower bounds of the decision variables. If the initial solution is an infeasible solution, a phase I optimization is initiated, which minimizes an objective function consisting of the sum of infeasibilities until a feasible point is found. Once this is achieved, the actual objective function 
replaces the sum of infeasibilities and the actual optimization phase is initiated. Using an initial point provided by the user allows the inclusion of engineering judgment in selecting a good initial solution which may or may not be feasible. In either case, experience has shown that a good user-provided initial point results in less computer time than initializing the algorithm with the variables at their lower bounds.

The algorithm for solving the chance constrained model starts with an initial solution with initial flow directions so that flows in the network are balanced. At each iteration of the search procedure within the generalized reduced gradient method, a check is made to determine if any $H_{i}$ is less than $H_{j}$ because of term $\left(H_{i}-H_{j}\right)^{0.54}$ in the constraints. If $H_{i}<H_{j}$, then $\mid\left(H_{i}-\right.$ $\left.H_{j}\right)\left.\right|^{0.54}$ is multiplied by -1 , indicating a change in flow direction. At the optimal solution, if there is any $\left(H_{i}-H_{j}\right)<0$, then $\left(H_{i}-H_{j}\right)$ is multiplied by -1 and $H_{i}$ and $H_{j}$ are interchanged. This procedure then updates the appropriate flow directions throughout the optimization iteration. Such a procedure does not cause any problems in convergence since the gradients are continuous.

\section{EXAMple Applications}

To illustrate the use of the model, two examples are included. The first is a simple hypothetical network, shown in Fig. 1, which has two loops and eight pipes, each $3,280 \mathrm{ft}$ in length, and mean demand at each node as shown in the figure. All the nodes are assumed to be at the same elevation and the pressure head at the source, node 1 , is $196.8 \mathrm{ft}$. The mean nodal pressure head requirement at each node is $100 \mathrm{ft}$, and the mean Hazen-Williams roughness coefficient is 100 for each link. The formulation, however, does

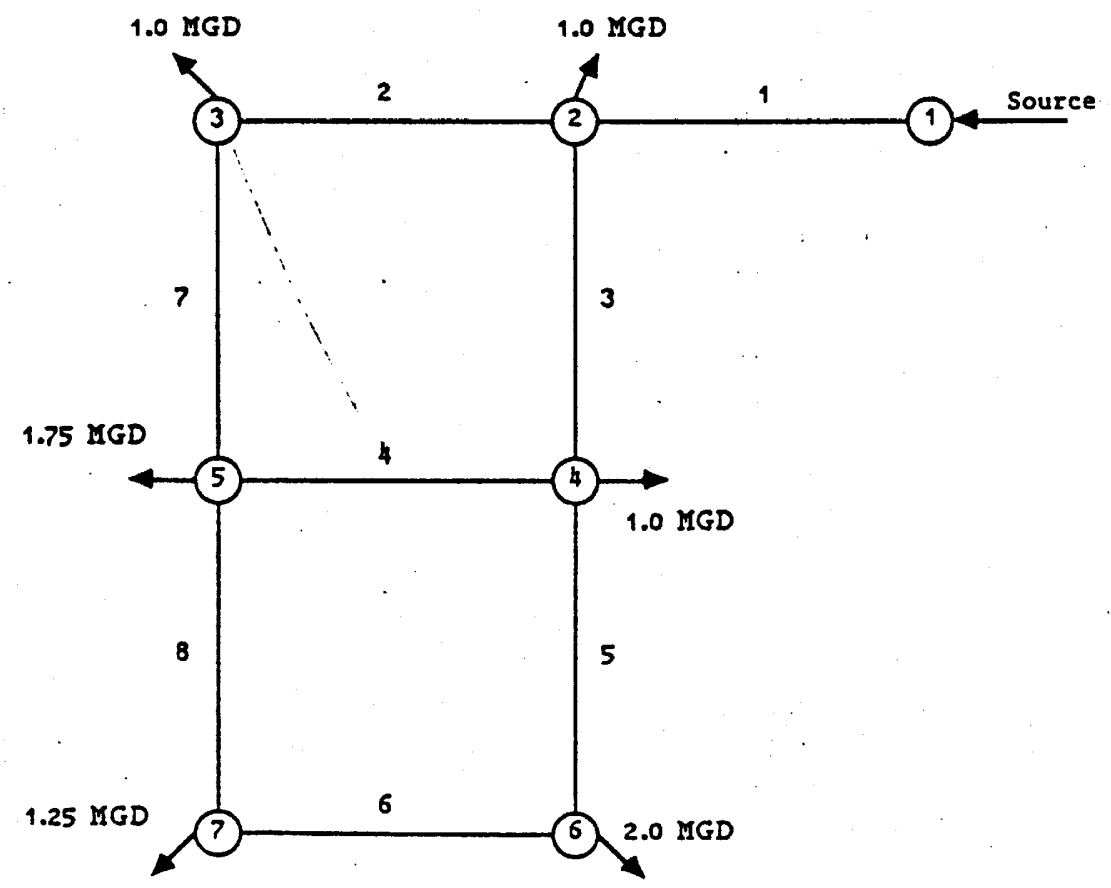

FIG. 1. Example 1: Elght PIpe Network 


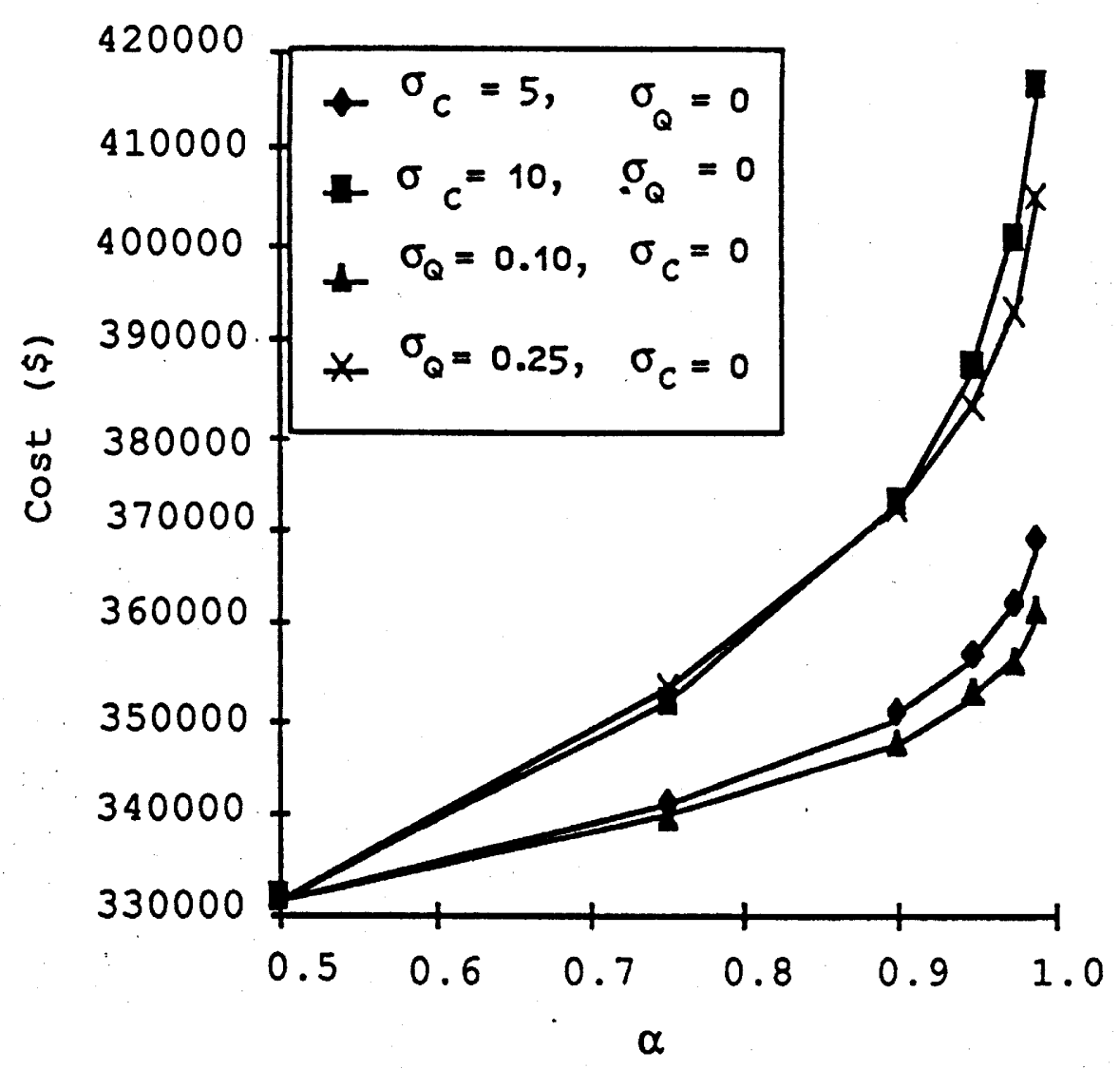

FIG. 2. Cost versus Rellabllity Requirement $\left(\sigma_{H}=0\right)$

not assume that all nodes have the same pressure head requirement or that they are on a level plane. Also, a general network with one or more source nodes can be incorporated, if desired. The cost of pipe for each link used in both examples is

$\operatorname{COST}=0.331 L D^{1.51}$

where $D=$ the pipe diameter in inches and $L=$ the length of the link in $\mathrm{ft}$. This equation is representative for thickness Class 23 cast iron pipe and includes the cost for purchasing, hauling, and laying (U.S. Army Corps of Engineers 1980). Any cost function, linear or nonlinear, could be used as Eq. 25 is used only for the purpose of illustrating the model application.

Several computer runs were made using various values of the standard deviation of the demand, pressure head, and roughness coefficient, in order to illustrate the impact of different levels of uncertainty on the design cost. The standard deviations selected for the nodal demands were $0.0,0.10$, and $0.25 \mathrm{mgd}$. Selected standard deviations for the pressure heads were 0,5 , and $10 \mathrm{ft}$ and for the Hazen-Williams roughness were 0,5 , and 10. A standard deviation equal to zero refers to the case of no uncertainty, and the larger the standard deviation, the greater the uncertainty. Computer runs were made for various values of $\alpha$ and $\beta$ ranging from 0.5 to 0.99 . Using $\alpha=$ $0.5(\beta=0.5)$ is equivalent to using mean values of the nodal demands and the pressure heads. Higher values of $\alpha$ and $\beta$ refer to more stringent per- 


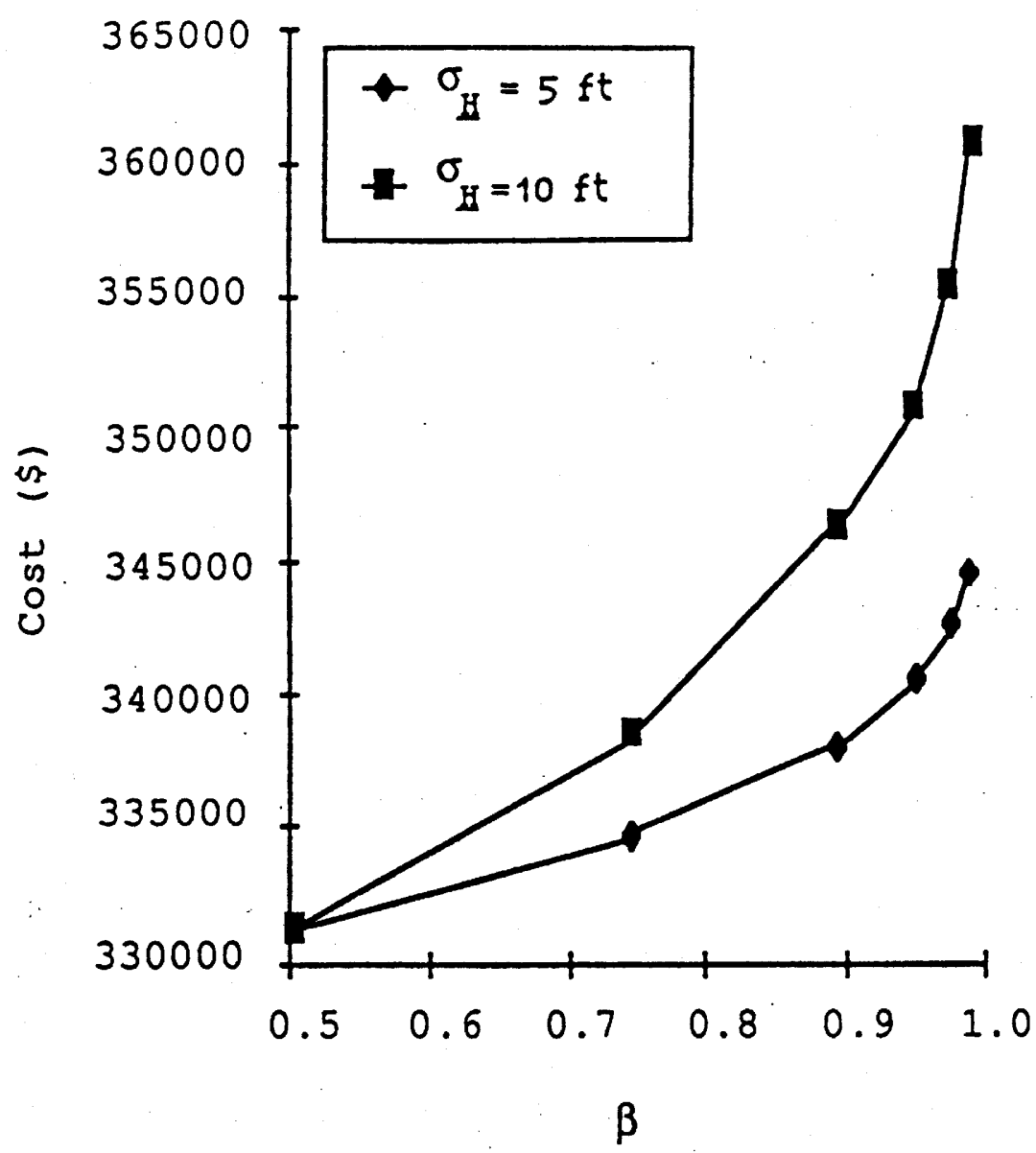

FIG. 3. Cost versus Pressure Head Rellability Requirement $\left(\sigma_{c}=\sigma_{Q}=0\right.$ )

formance requirements so that the likelihood of not meeting future demands and pressure head requirements is reduced.

Fig. 2 illustrates the impact of increasing the standard deviation of the roughness coefficient and the nodal demands, independently, while assuming the nodal pressure head requirement is known with certainty $\left(\sigma_{H}=0\right)$. With only Hazen-Williams roughness being uncertain $\left(\sigma_{H}=0\right.$ and $\left.\sigma_{Q}=0\right)$, as expected, the higher the reliability requirement, the greater the cost of the

TABLE 1. Example 1: Variability in Cost Due to Changing $\beta$ and Constant $\alpha$

\begin{tabular}{|c|c|c|c|c|}
\hline \multirow[b]{2}{*}{$\begin{array}{c}\beta \\
(1)\end{array}$} & \multicolumn{2}{|c|}{$\sigma_{c}=5$ and $\sigma_{Q}=0.10$} & \multicolumn{2}{|c|}{$\sigma_{c}=10$ and $\sigma_{c}=0.25$} \\
\hline & $\begin{array}{c}\sigma_{H}=5 \mathrm{ft}(\$) \\
(2)\end{array}$ & $\begin{array}{c}\sigma_{H}=10 \mathrm{ft}(\$) \\
(3)\end{array}$ & $\begin{array}{c}\sigma_{t}=5 \mathrm{ft}(\$) \\
(4)\end{array}$ & $\begin{array}{c}\sigma_{H}=10 \mathrm{ft}(\$) \\
(5)\end{array}$ \\
\hline 0.5 & 367,780 & 367,780 & 416,960 & 416,960 \\
\hline 0.75 & 371,810 & 376,060 & 421,560 & 426,400 \\
\hline 0.90 & 375,660 & 384,300 & 425,900 & 435,720 \\
\hline 0.95 & 378,030 & 389,610 & 428,610 & 441,700 \\
\hline 0.975 & 380,130 & 394,450 & 430,960 & 447,220 \\
\hline 0.99 & 383,680 & 400,470 & 433,800 & 453,960 \\
\hline
\end{tabular}


TABLE 2. Optimal Pipe Diameters for Example 1

\begin{tabular}{|c|c|c|c|c|c|c|}
\hline (a) & \multicolumn{3}{|c|}{$\sigma_{Q}=0.10$ and $\sigma_{H}=0.0$} & \multicolumn{3}{|c|}{$\sigma_{Q}=0.25$ and $\sigma_{H}=0.0$} \\
\hline$(\alpha, \beta)$ & $(0.5,0.5)$ & \multicolumn{2}{|r|}{$(0.99,0.5)$} & \multicolumn{2}{|c|}{$(0.99,0.5)$} & $(0.99,0.5)$ \\
\hline $\begin{array}{l}\text { Pipe } \\
\text { (1) }\end{array}$ & $\begin{array}{c}\sigma_{c}=5 \\
(2)\end{array}$ & \multicolumn{2}{|r|}{$\begin{array}{c}\sigma_{c}=5 \\
(3)\end{array}$} & \multicolumn{2}{|c|}{$\begin{array}{c}\sigma_{c}=5 \\
(4)\end{array}$} & $\begin{array}{c}\sigma_{c}=10 \\
(5)\end{array}$ \\
\hline $\begin{array}{c}1 \\
2 \\
3 \\
4 \\
5 \\
6 \\
7 \\
8 \\
\text { Cost (\$) }\end{array}$ & $\begin{array}{r}18.0 \text { in. } \\
6.8 \text { in. } \\
16.3 \text { in. } \\
9.2 \text { in. } \\
13.0 \text { in. } \\
9.9 \text { in. } \\
0.0 \text { in. } \\
0.0 \text { in. } \\
\$ 331,160\end{array}$ & \multicolumn{2}{|r|}{$\begin{array}{r}20.4 \text { in. } \\
7.4 \text { in. } \\
18.0 \text { in. } \\
9.9 \text { in. } \\
14.1 \text { in. } \\
10.7 \text { in. } \\
0.0 \text { in. } \\
0.0 \text { in. } \\
\$ 384,090\end{array}$} & \multicolumn{2}{|c|}{$\begin{array}{r}21.6 \text { in. } \\
8.1 \text { in. } \\
19.1 \text { in. } \\
10.4 \text { in. } \\
14.9 \text { in. } \\
11.4 \text { in. } \\
0.0 \text { in. } \\
0.0 \text { in. } \\
\$ 420,720\end{array}$} & $\begin{array}{r}23.6 \text { in. } \\
8.3 \text { in. } \\
20.3 \text { in. } \\
10.7 \text { in. } \\
15.4 \text { in. } \\
11.8 \text { in. } \\
0.0 \text { in. } \\
0.0 \text { in. } \\
\$ 458,730\end{array}$ \\
\hline (b) & \multicolumn{3}{|c|}{$\sigma_{Q}=0.10$ and $\sigma_{H}=5.0$} & \multicolumn{3}{|c|}{$\sigma_{Q}=0.25$ and $\sigma_{H}=10.0$} \\
\hline$(\alpha, \beta)$ & $(0.95,0.5)$ & \multicolumn{2}{|c|}{$(0.95,0.99)$} & \multicolumn{2}{|c|}{$(0.95,0.99)$} & $(0.95,0.99)$ \\
\hline Pipe & $\sigma_{c}=5$ & \multicolumn{2}{|r|}{$\sigma_{c}=5$} & \multicolumn{2}{|c|}{$\sigma_{c}=5$} & $\sigma_{c}=10$ \\
\hline $\begin{array}{c}1 \\
2 \\
3 \\
4 \\
5 \\
6 \\
7 \\
8 \\
\text { Cost (\$) }\end{array}$ & $\begin{array}{r}19.6 \text { in. } \\
7.2 \text { in. } \\
17.5 \text { in. } \\
9.7 \text { in. } \\
13.7 \text { in. } \\
10.4 \text { in. } \\
0.0 \text { in. } \\
0.0 \text { in. } \\
\$ 367,780\end{array}$ & \multicolumn{2}{|r|}{$\begin{array}{r}20.1 \text { in. } \\
7.4 \text { in. } \\
17.9 \text { in. } \\
10.0 \text { in. } \\
14.1 \text { in. } \\
10.7 \text { in. } \\
0.0 \text { in. } \\
0.0 \text { in. } \\
\$ 383,680\end{array}$} & \multicolumn{2}{|c|}{$\begin{array}{r}20.7 \text { in. } \\
7.7 \text { in. } \\
18.5 \text { in. } \\
10.3 \text { in. } \\
14.6 \text { in. } \\
11.0 \text { in. } \\
0.0 \text { in. } \\
0.0 \text { in. } \\
\$ 420,720\end{array}$} & $\begin{array}{r}23.0 \text { in. } \\
8.4 \text { in. } \\
20.1 \text { in. } \\
10.9 \text { in. } \\
15.6 \text { in. } \\
11.9 \text { in. } \\
0.0 \text { in. } \\
0.0 \text { in. } \\
\$ 453,960\end{array}$ \\
\hline (c) & \multicolumn{6}{|c|}{$\sigma_{H}=0.0$} \\
\hline Pipe & \multicolumn{2}{|c|}{$\sigma_{Q}=0.0$ and $\sigma_{c}=10$} & \multicolumn{2}{|c|}{$\sigma_{Q}=0.25$ and $\sigma_{c}=0$} & \multicolumn{2}{|c|}{$\sigma_{Q}=0.0$ and $\sigma_{c}=0$} \\
\hline$(\alpha, \beta)$ & \multicolumn{2}{|l|}{$(0.99,0.5)$} & \multicolumn{2}{|c|}{$(0.99,0.5)$} & & $(0.5,0.99)$ \\
\hline $\begin{array}{c}1 \\
2 \\
3 \\
4 \\
5 \\
6 \\
7 \\
8 \\
\text { Cost (\$) }\end{array}$ & \multicolumn{2}{|l|}{$\begin{array}{r}22.2 \text { in. } \\
7.4 \text { in. } \\
19.2 \text { in. } \\
10.1 \text { in. } \\
14.7 \text { in. } \\
10.8 \text { in. } \\
0.0 \text { in. } \\
0.0 \text { in. } \\
\$ 416,650\end{array}$} & \multicolumn{2}{|c|}{$\begin{array}{r}20.6 \text { in. } \\
8.2 \text { in. } \\
18.4 \text { in. } \\
10.3 \text { in. } \\
14.7 \text { in. } \\
11.3 \text { in. } \\
0.0 \text { in. } \\
0.0 \text { in. } \\
\$ 404,360\end{array}$} & & $\begin{array}{r}19.0 \text { in. } \\
7.2 \text { in. } \\
17.2 \text { in. } \\
9.8 \text { in. } \\
13.7 \text { in. } \\
10.4 \text { in. } \\
0.0 \text { in. } \\
0.0 \text { in. } \\
\$ 360,630\end{array}$ \\
\hline
\end{tabular}

design. The same is true for different standard deviations of nodal demand and known Hazen-Williams roughness coefficient $\left(\sigma_{H}=0\right.$ and $\left.\sigma_{C}=0\right)$. Fig. 3 shows the change in cost with increasing $\beta$ for two standard deviations of nodal pressure and no uncertainty in the nodal demands or roughness coefficients $\left(\sigma_{c}=0, \sigma_{Q}=0\right)$. The same trend is apparent in this figure as seen in Fig. 2. Table 1 provides the system costs for different values of the standard deviations and levels of $\alpha$ and $\beta$.

Table 2 lists the optimal designs for selected values of the standard de- 


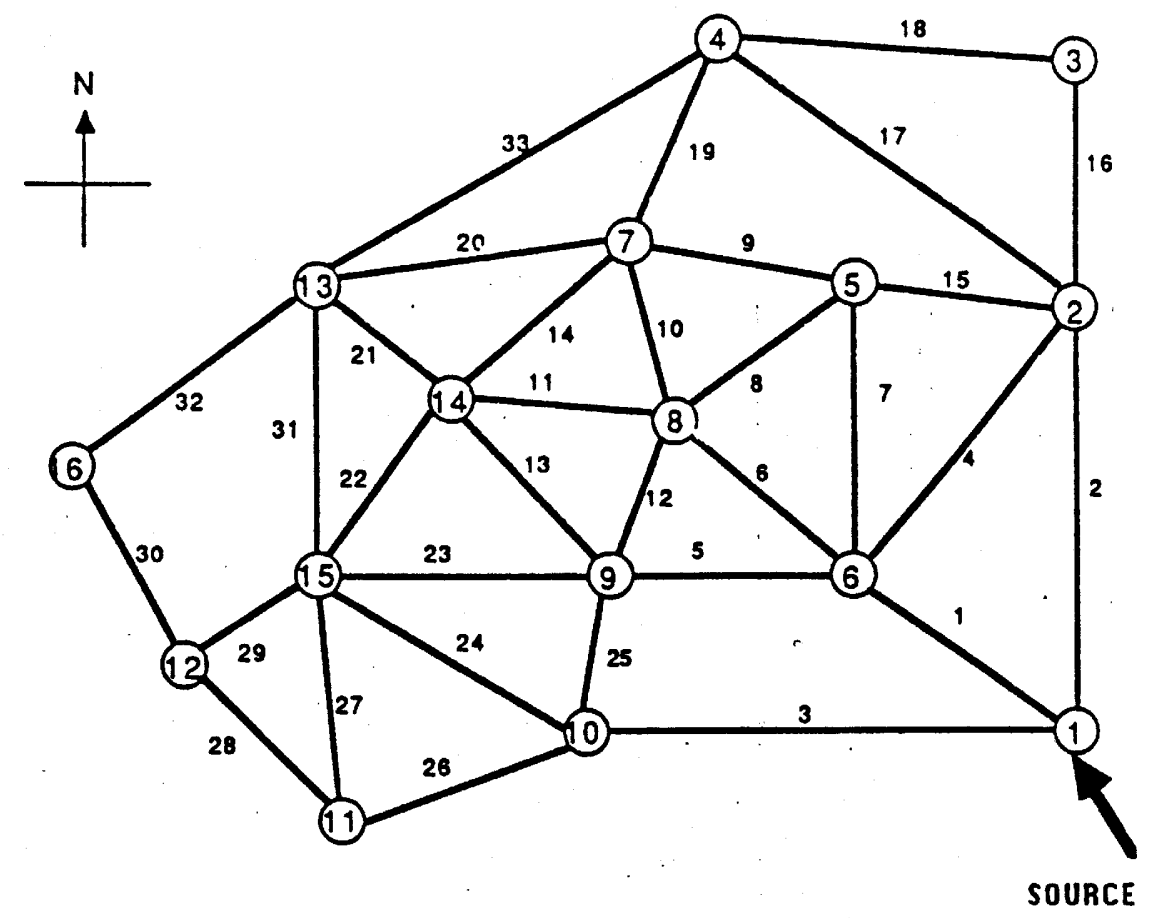

(not to scale)

FIG. 4. Example 2 Network

viation to show the changes in the system for different reliability requirements. The optimal continuous diameters could be converted to discrete diameters by considering them as equivalent pipe diameters and determining the lengths of two pipes which make up the link and have the same hydraulic characteristics. All of the optimal solutions were branched networks as expected for the optimal design of systems under a single demand pattern. The nonlinear programming problem for this example consisted of 16 decision variables, 6 nonlinear constraints, and 14 simple bounds. The computation time required to determine a design was usually about 2 seconds of CPU time on the University of Texas Dual Cyber 170/750.

The second application considered a more realistic size network consisting of 33 pipes and 16 nodes (Fig. 4) with the pipe lengths listed in Table 3.

TABLE 3. Plpe Lengths for Example 2

\begin{tabular}{c|c}
\hline \hline $\begin{array}{c}\text { Pipe number } \\
(1)\end{array}$ & Length (ft) \\
\hline $1-3$ & 12,000 \\
4 & 9,000 \\
$5-16$ & 6,000 \\
17 & 9,000 \\
$18-26$ & 6,000 \\
27 & 9,000 \\
$28-31$ & 6,000 \\
$32-33$ & 12,000 \\
\hline
\end{tabular}


TABLE 4. Optimal Solution (Plpe Dlameters) for Different $\alpha$ and $\beta$ for Example 2

\begin{tabular}{|c|c|c|c|}
\hline \multirow[b]{2}{*}{$\begin{array}{l}\text { Pipe } \\
\text { (1) }\end{array}$} & \multicolumn{3}{|c|}{ Pipe Diameters (in.) } \\
\hline & $\begin{array}{c}\alpha=\beta=0.5 \\
(2)\end{array}$ & $\begin{array}{c}\alpha=\beta=0.75 \\
\text { (3) }\end{array}$ & $\begin{array}{c}\alpha=\beta=0.90 \\
\text { (4) }\end{array}$ \\
\hline 1 & 22.8 & 10.4 & 24.2 \\
\hline 2 & 18.8 & 21.7 & 15.9 \\
\hline 3 & 20.9 & 30.0 & 31.4 \\
\hline 4 & 0.0 & 0.0 & 0.0 \\
\hline 5 & 0.0 & 0.0 & 11.4 \\
\hline 6 & 21.5 & 0.0 & 11.4 \\
\hline 7 & 0.0 & 0.0 & 16.4 \\
\hline 8 & 0.0 & 0.0 & 0.0 \\
\hline 9 & 0.0 & 0.0 & 12.9 \\
\hline 10 . & 15.3 & 0.0 & 0.0 \\
\hline 11 & 10.5 & 0.0 & 0.0 \\
\hline 12 & 10.5 & 11.7 & 0.0 \\
\hline 13 & 0.0 & 11.7 & 0.0 \\
\hline 14 & 0.0 & 0.0 & 0.0 \\
\hline 15 & 10.0 & 10.5 & 0.0 \\
\hline 16 & 10.0 & 10.5 & 12.7 \\
\hline 17 & 11.5 & 14.5 & 0.0 \\
\hline 18 & 0.0 & 0.0 & 0.0 \\
\hline 19 & 0.0 & 12.9 & 1.3 \\
\hline 20 & 12.5 & 0.0 & 0.0 \\
\hline 21 & 0.0 & 0.0 & 14.1 \\
\hline 22 & 0.0 & 0.0 & 0.0 \\
\hline 23 & 0.0 & 0.0 & 0.0 \\
\hline 24 & 9.6 & 21.5 & 28.9 \\
\hline 25 & 0.0 & 18.2 & 0.0 \\
\hline 26 & 17.5 & 10.2 & 0.0 \\
\hline 27 & 0.0 & 0.0 & 12.8 \\
\hline 28 & 15.7 & 0.0 & 0.0 \\
\hline 29 & 0.0 & 16.6 & 17.6 \\
\hline 30 & 12.8 & 13.4 & 14.1 \\
\hline 31 & 0.0 & 11.2 & 17.6 \\
\hline 32 & 0.0 & 0.0 & 0.0 \\
\hline 33 & 0.0 & 0.0 & 0.0 \\
\hline Cost & $\$ 2,144,300$ & $\$ 2,258,400$ & $\$ 2,613,533$ \\
\hline
\end{tabular}

This system was also assumed to be on a level plane although this restriction is not necessary since the pressure head requirement may be different for each node. The demand is assumed to be uniform throughout the system with a 1 mgd mean demand at each node. The pipe roughness has a mean of 130 for all pipes and the mean minimum pressure head requirement is $92.3 \mathrm{ft}$ at every node. The pressure head at the source node was fixed at $135.0 \mathrm{ft}$. The model was executed for values of $a$ and $\beta$ equal to $0.5,0.75$, and 0.90 , with the pipe diameters and total costs listed in Table 4. As in the previous example, the cost of the system increases with the reliability requirement. The networks for $\alpha, \beta=0.5,0.75$ and 0.90 are presented in 


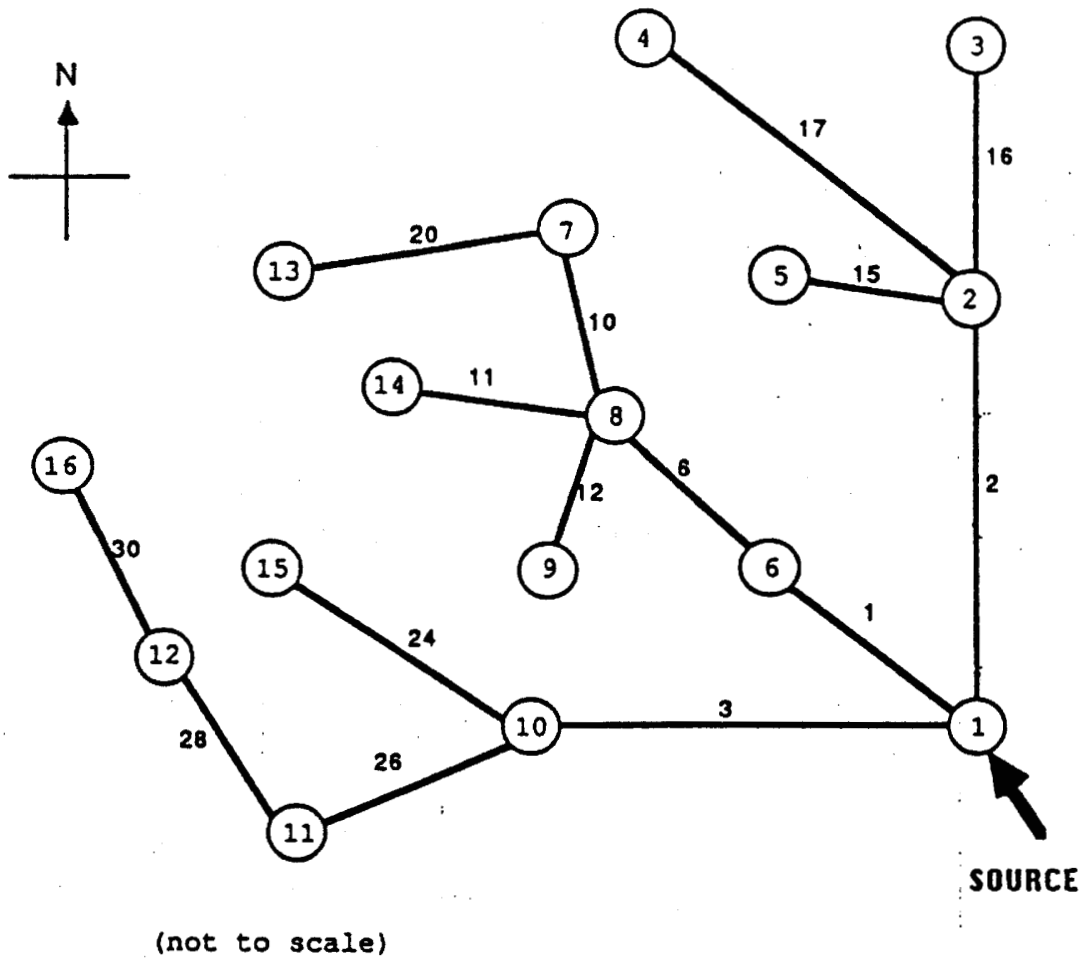

FIG. 5. Optimal Network for $(\alpha=\beta=0.5)$
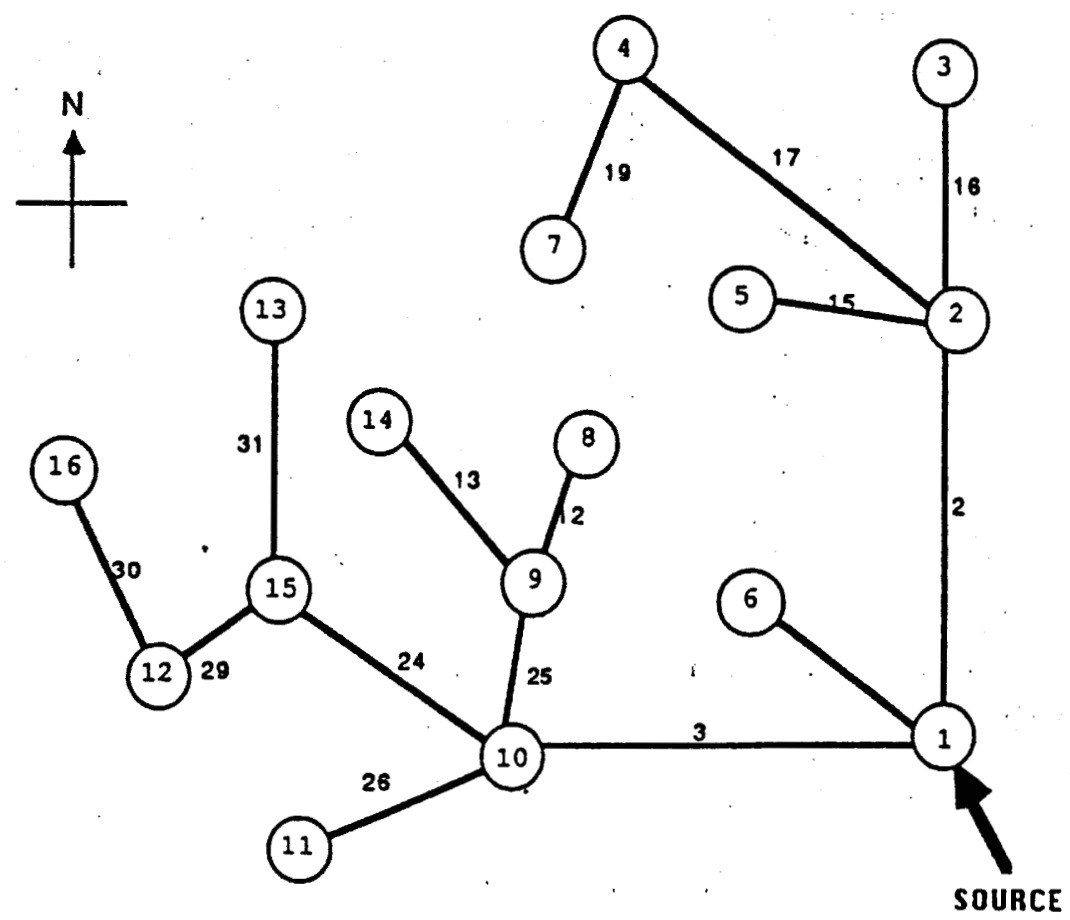

(not to scale)

FIG. 6. Optimal Network for $(\alpha=\beta=0.75)$ 


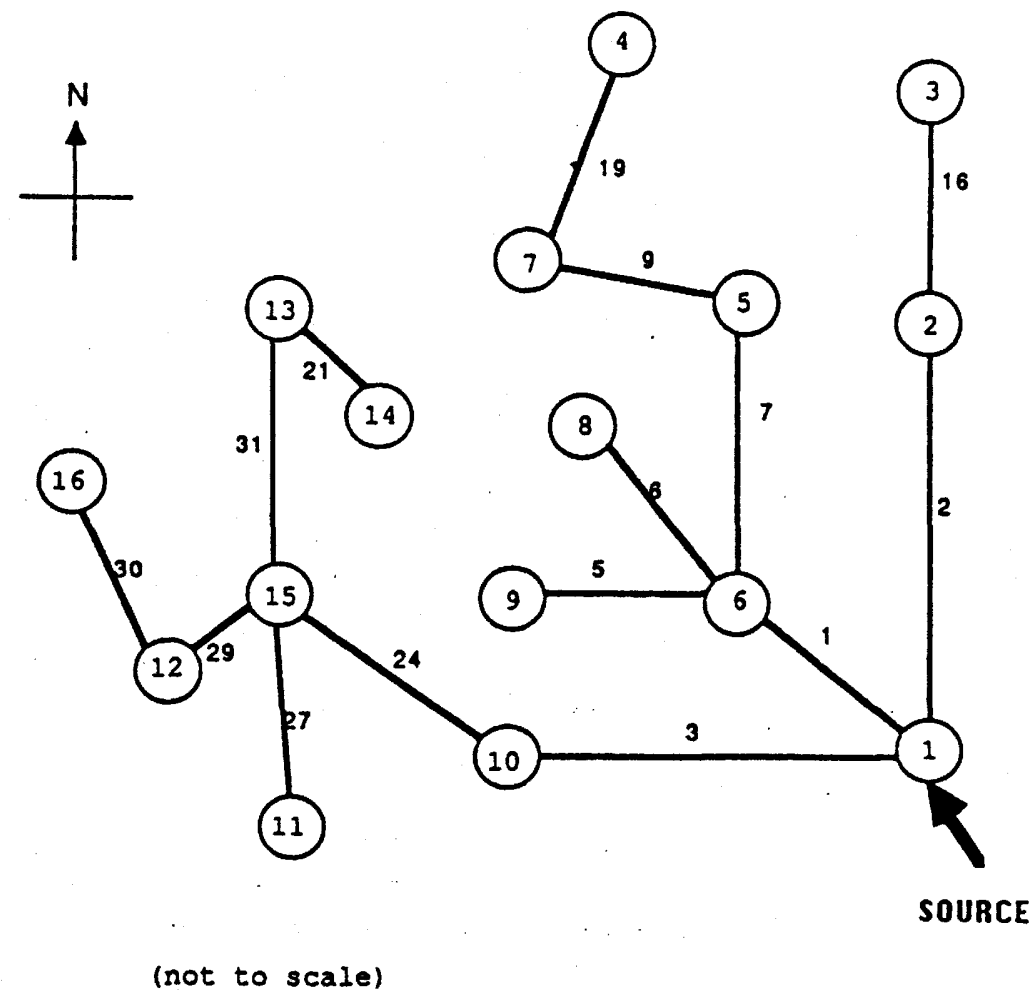

FIG. 7. Optlmal Network for $(\alpha=\beta=0.90)$

Figs. 5, 6, and 7, respectively. It is interesting to point out that the variation of constraint performance reliability not only affects the total system cost but also results in different network configurations.

The nonlinear programming problem for this example consisted of $49 \mathrm{de}-$ cision variables, 16 nonlinear constraints, and 49 simple bounds. The computation time required for a typical problem was $100 \mathrm{CPU}$ seconds on the University of Texas Dual Cyber system. In solving the model, the gradients in GRG2 were computed by the numerical finite difference scheme although, based on experience with other problems, analytically calculating the gradients of the constraints would be expected to reduce computation time by roughly $20-25 \%$.

\section{Conclusions}

Two observations that might have important implications in design can be made from the above analysis. First, the cost versus reliability relationship is convex. Thus, to increase the $a$ or $b$ an incremental amount at a higher reliability level will result in a greater increase in the system cost than for an incremental change at a lower level. The trade-off decision between the level of confidence desired in the design and cost becomes more complex in terms of deciding what is cost effective.

The second observation from the first example is that in this case it is more costly to achieve higher levels of confidence in the nodal demands than for the nodal pressures. This, unfortunately, is the opposite of what is known in practice when designing a system. A requirement for the desired 
nodal pressure can be set with a fair level of confidence. However, to accurately estimate what the demand will be at a particular node in the system or what the roughness in a link will be in the future is quite difficult.

The purpose of this paper has been to present a methodology for the minimum cost design of water distribution systems but to incorporate the various uncertainties explicitly into the design. In order to solve the nonlinear optimization problem, a generalized reduced gradient procedure was used. A global optimum cannot be guaranteed because of the nonlinearity and concavity of the problem. The GRG2 code was used to solve the problem; however, other available nonlinear programming codes could be used. The methodology also results in solutions with nondiscrete pipe diameters. Integer nonlinear programming solution techniques are not advanced to the point that they can be used. The investigators feel that rounding to commercial pipe sizes or converting the continuous, which would be considered as an equivalent, to two commercial pipe sizes for the link should not distract from use of the methodology.

The inherent uncertainties associated with the nodal demands, pipe roughness coefficients, and pressure head requirements have been considered in a methodology for the design of a water distribution system. By applying chance constrained programming techniques, a nonlinear optimization model has been formulated and solved for two example systems. The incorporation of the uncertainties into the design procedure results in a more reliable design than would be determined using an average condition. Since the pipe sizes are selected by an optimization procedure, the least cost design is determined for a specified reliability requirement. By varying this requirement, decision makers can determine the trade-off between reliability and cost which would lead to more informed and better decisions.

\section{ACKNOWLEDGMENTS}

This material is based upon the research project, "Assessment of Aging Water Systems;" supported by the National Science Foundation Grant No. ECE-8511399, under the direction of Larry W. Mays.

\section{Appendix. References}

Alperovits, E., and Shamir, U. (1977). "Design of optimal water distribution systems." Water Resour. Res., 13(6), 885-900.

Charnes, A., and Cooper, W. (1963). "Deterministic equivalents for optimizing and satisfying under chance constraints." Operations Res., 11(1), 18-39.

Charnes, A., and Sterdy, A. C. (1966). "A chance-constrained model for real-time control in research and development management." Mgmt. Sci., 12(8), B-353 to B-363.

Coals, A., and Goulter, I. C. (1985). "Approaches to the consideration of reliability in water distribution networks." Proc., 1985 International Symp. on Urban Hydrology, Hydraulic Infrastructures and Water Quality Control, Univ. of Kentucky; Lexington, Ky., 287-295.

Hudson, W. D. (1966). "Studies of distribution system capacity in seven cities." J. Am. Water Works Assoc., 157-164.

Jeppson, R. W. (1976). Analysis of flow in pipe networks. Ann Arbor Science, Ann Arbor, Mich.

Lansey, K. E., and Mays, L. W. (1987). "Optimal design of large scale water distribution systems." Proc., 1987 National Conference on Hydraulic Engineering, ASCE, Williamsburg, Va., 475-480. 
Lasdon, L. S., and Waren, A. D. (1984). GRG2 user's guide. Department of General Business Administration, The University of Texas at Austin, Austin, Tex.

Mays, L. W., and Cullinane, M. J. (1986). "A review and evaluation of reliability concepts for design of water distribution systems." Miscellaneous Paper EL-861, U.S. Army Corps of Engineers, Waterways Experiment Station, Vicksburg, Miss.

Morgan, D. R., and Goulter, I. (1985). "Optimal urban water distribution design." Water Resour. Res., AGU, 21(5).

Quindry, G. E., Brill, E. D., and Liebman, J. C. (1981). "Optimization of looped water distribution systems." J. Envir. Engrg. Div., ASCE, 197(4), 665-679.

Su, Y.-C., et al. (1987). "Reliability based optimization model for water distribution systems." J. Hydr. Engrg., ASCE, 114(12), 1539-1556.

U.S. Army Corps of Engineers. (1980). "Methodology for areawide planning studies." Engineer Manual 111-2-502, Washington, D.C. 